\title{
TRANSFER EFFECT OF $N$-BACK TRAINING: MATHEMATICAL IMPLICATIONS IN SCHOOL-AGE CHILDREN
}

\author{
Selma Boz \\ Department of Education, Eötvös Loránd University (Hungary)
}

\begin{abstract}
Working memory (WM) is the system which is used for short-term storage and where information about cognitive tasks is manipulated. One of the most important characteristics of WM is its limited capacity, which restricts cognitive performance. Despite of this limit in WM capacity, the efficiency of WM can be improved with WM training and this training effect can be transferred to performance on complex tasks, such as mathematical operations. Such training tasks are complex and necessarily include core processes and these measures, therefore, contribute to difficulty to design tasks and interpret the outcomes for specific changes gained from the training. For example, $n$-back tasks which are used in a wide range of research are based on core training. Since core trainings address the executive functions of WM and enhance the domain-general aspects, increasing performance on domain-general factors may promote both near and far transfer effects of training. In the current study, WM training will be constructed on the basis of the interference framework that characterizes individual differences in WM performance. The aim of this study is to explore individual differences in training and the way transfer effects occur, evaluating gains from Mathematics proficiency. An adaptive version of $n$-back tasks will be implemented for the proposed study, within WM load and interference lures. The study will be carried out with 40 school-age children between the ages of 9 and 12, and Solomon four group design method will be used to group them. $d$ ' (D-Prime) theory will be conducted in order to obtain detailed comparison between groups as well as interpretation of individual differences in processing of information.
\end{abstract}

Keywords: Working memory, n-back training, near transfer effect, far transfer effect, mathematics.

\section{Introduction}

The complex tasks in Mathematics (Math) include numerous cognitive processes and strategies which are necessary for Math skills. Accordingly, cognitive processes related to numbers and counting abilities are identified with working memory (WM) (Hubber et al., 2014) where information is stored and monitored, as well as manipulated (De Stefano \& LeFevre, 2004; Raghubar, Barnes, \& Hecht, 2010). However, the prominent feature of WM is its limited capacity (Baddeley, 2000). Therefore, a task can contribute to exceeding individual's available capacity (Just \& Carpenter, 1992), leading to poor performance in Math.

The main theoretical framework of this study has been offered to describe the basis of individual differences in WM capacity and to explain the functions of WM. In this framework, this paper presents a possible outcomes of how interference theory (Cowan, 2000; Oberauer, 2001) defines individual differences in WM performance along with $n$-back training and how trained, process-specific improvements in WM performance may affect mathematical improvements in school-age learners as transfer effects.

As a result of the training outcomes, this study may help curriculum designers to immerse training programs in Math curriculum to create more efficient teaching programs regarding children's individual differences.

\section{Theoretical background}

\subsection{Working memory}

It is conceded that working memory (WM) is a system which has a processing-oriented construct and supplies active processing and temporary storage of task-relevant information (Cowan, 1995; Oberauer, 2009). One of the most important characteristics of WM is its limited capacity, which contributes to restriction in cognitive performance. WM capacity is associated with performance on cognitive tasks. For instance, performance of individuals with greater capacity might be better than individuals with lesser 
capacity in cognitive domains (Conway, Jarrold, Kane, Miyake \& Towse, 2007). In accordance, understanding what constrains WM capacity is an important step toward understanding the reasons of individual differences in cognitive activities (Oberauer, Farrell, Jarrold \& Lewandowsky, 2016).

\subsection{Interference framework}

The limitation in WM capacity can be explained by interference between items and processes (Nairne, 1990; Oberauer \& Kliegl, 2006; Oberauer, 2009). According to this interference theory, WM is a limited-capacity system, but this limitation is ascribed to interference among memory representations and processes (Oberauer, 2009). In brief, when an individual faces a flow of information, his/her limit of capacity can be exceeded. This exceeding in capacity might lead to limitations to hold information in memory and to update those items during the processing of new information. It eventually becomes difficult to differentiate old information from the new one. The capability to resist interferences is required to accomplish complex tasks (Nigg, 2000). Here, interference control can provide a link between WM and information processing in Math. As an example, regarding Math settings, it is possible that interference will occur if a given problem is largely similar to a previously learned problem, resulting in all likelihood less retrieval capability (De Visscher \& Noël, 2014). Therefore, the efficiency of memory representations of Math problems, especially arithmetical problems, depends on previously learned problems (i.e. overlap theory) (Nairne, 1990).

Consequently, poor performance in WM is based on inadequate control of irrelevant information (Hasher and Zacks, 1988). It becomes unavoidable to recall irrelevant information instead of target items, if no longer relevant items cannot be suppressed (Palladino, 2006). On that occasion, while an individual is processing information, these relevant and irrelevant items induce competing for limited access in WM. $\mathrm{He} / \mathrm{she}$ must resist irrelevant information to remove them once they get access or give access in the first place in memory (Hasher, Lustig \& Zack, 2007; Unsworth \& Engle, 2007). Individuals who can manage this process with their skills of interference control perform better on higher-order cognitive tasks than individuals with poor control abilities (Unsworth, 2010; Friedman \& Miyake, 2004). Therefore, interference control is an essential construct of individual differences for cognitive abilities.

\section{3. $n$-Back training and transfer effects}

It is propounded that the efficiency of WM can be improved with WM training (Klingberg et al., 2005; Verhaeghen, Cerella, \& Basak, 2004; Westerberg et al., 2007). Due to the fact that WM plays a key role in various processes, as well as individual differences in cognitive performance, the main target about training studies is to recruit WM mechanism and transfer training effect to performance on complex tasks (Sternberg, 2008).

The decision about whether the training is effective depends on its transfer effects on to untrained tasks (Shipstead, Redick, \& Engle, 2010). Accordingly, WM training is considered to provide both near and far transfer effects (Barnett \& Ceci, 2002). Near transfer effect concerns with improvement in a related domain, such as WM scores, while far transfer effect relies on a measure that training can affect other related skills. Near transfer effect concerns with improvement in a related domain, such as WM scores, while far transfer effect is based on a measure that training can affect other related skills. The outcome of far transfer effect is significant to predict the efficiency of a training program, however near transfer effect might be devalued as a structure of practice effect if near transfer tests reflect similar outcomes to the training tasks (Klingberg et al., 2005). Additionally, the methods of WM training generally can be classified as "core training" and "strategy training", as revealed by Morrison and Chein (2011). This categorization is based on training targeting that serves either domain-general or domain-specific aspects of WM. Core-based training paradigms are conceived to target domain-general mechanisms and precisely aimed to embrace overall functions, such as maintaining, encoding and retrieval of information, not a specific type of information. Such training tasks are complex and necessarily includes core processes and these measures, therefore, contribute to difficulty to design tasks and interpret the outcomes for specific changes gained from the training. Since core trainings target executive functions of WM and enhance the domain-general aspects, increasing performance on domain-general factors may promote both near and far transfer effects of training. For example, $n$-back tasks which are used in wide range of research are based on core training.

In an $n$-back task, an examinee is subjected to stimuli such as letters or shapes appeared one on a computer screen at a limited time. In this task, the target is to decide whether the current item matches the one that was presented " $n$ " items prior. " $n$ " can be manipulated to increase or decrease the load in the WM system. During the task, the examinee needs to make a recognition decision on each item by accepting targets and rejecting distractors based on the $n$-back rule (Oberauer et al., 2007). In this manner, information is presented rapidly and there are only " $n$ " number of relevant temporal positions, incoming items are bound to the same temporal context as previous items and the WM system is required to resist interference from this previously relevant information. 


\section{Methodology}

The research will include 40 Turkish students in primary schools, between the ages of 9 and 12 years. These participants will be randomly divided into four groups, according to the Solomon 4-group design, the strongest design for treatment studies, and each group will be engaged in a different combination of experimental manipulations: the first group will receive the pre-test, the training, and the post-test; the second group will receive only the training and the post-test; the third group will receive the pre-test, no training, and the post-test, and the forth group will receive only the post-test. The training groups will receive 16 online training sessions.

To address the aim of the study related to differences in transfer of trained skills to Math tasks between the experimental and control groups, accuracy and reaction time will be collected from the adaptive $n$-back paradigm and Math proficiency tests via E-Prime Go Software. The experimental groups will be completing an adaptive $n$-back training with lures, which were developed in the Cognition and Language Laboratory at the Graduate Center of the City University of New York (Wadhera, Campanelli, \& Marton, 2018). Within these groups, effects of individual differences and effects of experimental manipulations of interference and set size level on $n$-back performance will be analyzed. Signal Detection Theory will be used by calculating $d$ ' regarding $n$-back performance over the course of training. Between the experimental and control groups, three categories of Math tests will be administered before and after the training period to determine transfer effects. Performance of two control groups will be compared to that of the experimental groups.

A battery of Math proficiency tests will be administered online to participants in a randomized order, with respect to their group assignments by using E-Prime Go software. Three different tests will be used to examine participants' Math proficiency: WM updating test, arithmetic test and word problem test. The purpose of this task is to measure far transfer of trained skills of interference control to performance on Math tasks. Firstly, a standardized Math proficiency measure will be used, that includes the WM updating test (Pelegrina et al., 2020) to assess children's abilities to solve arithmetic problems while storing previous results for future use in calculation. A series of arithmetical operations appear consecutively in two boxes across all tasks. Depending on the task, participants are required to retrieve information associated with the box, apply the operation and then substitute the result for the corresponding box. Secondly, the arithmetic test will be designed based on interference theory, in addition to the principles of competition and cooperation among neighboring arithmetic problems (Vergut \& Fias, 2005). For instance, doing operations with multi-digit numbers needs holding intermediate results (e.g. holding tens in mind and doing carry operation) to attain a correct answer. Lastly, the word problem test (Ng, Lee and Khng, 2017) will be developed according to interference theories, that will embrace irrelevant information which participants will not need while solving the problem. Here, the participants will encounter distracting or competing information while trying to maintain the task goal and their performance in differentiating relevant and irrelevant information will be evaluated for this task.

\section{Expected outcomes and limitations}

In this study, it is a key aspect to determine whether this training will improve the WM system, which are related to manipulation of memory representations and to resisting interference during processing, and to understand how this improvement can be transferred to participants' Math skills. The possible outcomes are described based on these aims.

The experimental training group will show more pre- to post-training improvement than the control group on the numerical operations of Math proficiency. Training will contribute to gains in a population of elementary level children with different levels of arithmetic ability (Kuhn and Holling, 2014). Additionally, retrieval processes during Math learning allows consideration of the benefits of an interference-based WM training to children's performance on Math.

The second set of expected outcomes will address the differences across groups on described transfer tasks. Participants in both training groups will perform significantly better on the interference condition than the two control groups. They will also show significantly better performance on numerical operation tasks than the two control groups, which will contribute to strong evidence for the role of WM interference control in Mathematical abilities in school-age children. Alternatively, if the training groups show similar performance with the control groups, this may reveal that training domain-general WM mechanisms is not enough and that Math-specific processes have to be trained as well in order to improve performance on Math tasks. In addition, if the training groups perform significantly better on numerical operation tasks than the control groups, this will mean that binding and updating mechanisms play a crucial role in interference control and also ability in Math. Lastly, it is expected that the training groups and the control groups perform similarly on the specific tasks of fractions, decimals and algebra because 
I do not expect immediate effects of the WM training on a complex Math task that incorporates several other mechanisms, such as task difficulties and Mathematical prerequisites.

In this study, data collection would have happened during the 2020 fall semester in elementary schools in Turkey. Each participant would be tested and trained in person within the control of the investigator at their schools. Due to COVID-19, the schools were closed in Turkey where all task completion was planned to be done and the data could not be collected during the planned period. Therefore, the original plan was adapted to online data collection, which the process has not started yet.

\section{References}

Baddeley, A. (1992). Working memory. Science, 255, 556-559.

Barnett, S. M., \& Ceci, S. J. (2002). When and where do we apply what we learn? A taxonomy for far transfer. Psychological Bulletin, 128, 612-637.

Conway, A. R. A., Jarrold, C., Kane, M. J., Miyake, A., \& Towse, J. N. (Eds.). (2007). Variation in working memory. New York: Oxford University Press.

Cowan, N. (1995). Oxford psychology series, No. 26. Attention and memory: An integrated framework. New York, NY, US: Oxford University Press.

Cowan, N. (2000). Processing limits of selective attention and working memory: Potential implications for interpreting. Interpreting, 5(2), 117-146.

De Stefano, D., \& LeFevre, J-A. (2004). The role of working memory in mental arithmetic. European Journal of Cognitive Psychology, 16(3), 353-386.

De Visscher, A., Noël, M.-P., (2014). The detrimental effect of interference in multiplication facts storing: typical development and individual differences. J. Exp. Psychol.: General, 143, 2380-2400.

Friedman, N. P., \& Miyake, A. (2004). The relations among inhibition and interference control functions: A latent-variable analysis. Journal of Experimental Psychology: General, 133, 101-135.

Hasher, L., \& Zacks, R. T. (1988). Working memory, comprehension and aging: A review and new view. In G. H. Bower (Ed.), The psychology of learning and motivation. San Diego, CA: Academic Press.

Hasher, L., Lustig, C., \& Zacks, R. (2007). Inhibitory mechanisms and the control of attention. In A. R. A. Conway, C. Jarrold, M. J. Kane (Eds.) \& A. Miyake \& J. N. Towse (Ed.), Variation in working memory (p. 227-249). Oxford University Press.

Hubber, P.J., Gilmore, C. \& Cragg, L. (2014) The roles of the central executive and visuospatial storage in mental arithmetic: A comparison across strategies. The Quarterly Journal of Experimental Psychology, 67(5), 936-954.

Just, M. A., \& Carpenter, P. A. (1992). A capacity theory of comprehension: Individual differences in working memory. Psychological Review, 99(1), 122-149.

Klingberg, T., Fernell, E., Olesen, P. J., Johnson, M., Gustafsson, P., Dahlstrom, K., et al. (2005). Computerized training of working memory in children with ADHD-a randomized, controlled trial. Journal of the American Academy of Child and Adolescent Psychiatry, 44(2), 177-186.

Kuhn, J.-T., \& Holling, H. (2014). Number sense or working memory? The effect of two computer-based trainings on mathematical skills in elementary school. Advances in Cognitive Psychology, 10(2), 59-67.

Morrison, A. B., \& Chein, J. M. (2011). Does working memory training work? The promise and challenges of enhancing cognition by training working memory. Psychonimic Bulletin \& Review, 18, 46-60.

Nairne, J. S. (1990). A feature model of immediate memory. Memory \& Cognition, 18, 251-269.

$\mathrm{Ng}$, J., Lee, K., \& Khng, K. H. (2017). Irrelevant information in math problems need not be inhibited: Students might just need to spot them. Learning and Individual Differences, 60, 46-55.

Nigg, J. T. (2000). On inhibition/disinhibition in developmental psychopathology: Views from cognitive and personality psychology and a working inhibition taxonomy. Psychological Bulletin, 126(2), $220-246$

Oberauer, K. (2001). Removing irrelevant information from working memory: A cognitive aging study with the modified Sternberg task. Journal of Experimental Psychology: Learning, Memory, and Cognition, 27(4), 948-957.

Oberauer, K. (2009). Design for a working memory. In B. H. Ross (Ed.), The psychology of learning and motivation: Vol. 51. The psychology of learning and motivation (pp. 45-100). San Diego, CA, US: Elsevier Academic Press.

Oberauer, K., \& Kliegl, R. (2006). A formal model of capacity limits in working memory. Journal of Memory and Language, 55(4), 601-626. 
Oberauer, K., Farrell, S., Jarrold, C., \& Lewandowsky, S. (2016). What limits working memory capacity? Psychological Bulletin, 142(7), 758-799.

Oberauer, K., Süß, H.-M.,Wilhelm,O., and Sander, N.(2007).Individual differences in working memory capacity and reasoning ability, in A. R. A. Conway, C. Jarrold, M.J. Kane, A. Miyake and J. N. Towse (Eds), Variation in Working Memory, (pp. 49-75), New York, NY: Oxford University Press

Palladino, P. (2006). The Role of Interference Control in Working Memory: A Study with Children at Risk of Adhd. Quarterly Journal of Experimental Psychology, 59(12), 2047-2055.

Pelegrina, S., Justicia-Galiano, M.J., Martín-Puga, M.E. and Linares, R. (2020). Math Anxiety and Working Memory Updating: Difficulties in Retrieving Numerical Information from Working Memory. Front. Psychol. 11(669).

Raghubar, K. P., Barnes, M. A., \& Hecht, S. A. (2010). Working memory and math: A review of developmental, individual difference, and cognitive approaches. Learning and Individual Differences, 20 (2), 110-122.

Shipstead, Z., Redick, T. S., \& Engle, R. W. (2010). Does working memory training generalize? Psychologica Belgica, 50, 245-276.

Sternberg, R. J. (2008). Increasing fluid intelligence is possible after all. Proceedings of the National Academy of Sciences, 105(19), 6791-6792.

Unsworth, N. (2010). Interference control, working memory capacity, and cognitive abilities: A latent variable analysis. Intelligence, 38(2), 255-267.

Unsworth, N., \& Engle, R. W. (2007). The nature of individual differences in working memory capacity: Active maintenance in primary memory and controlled search from secondary memory. Psychological Review, 114, 104-132.

Verguts, T. \& Fias, W. (2005). Interacting neighbors: A connectionist model of retrieval in single-digit multiplication. Mem Cognit. 33(1), 1-16.

Verhaeghen, P., Cerella, J., \& Basak, C. (2004). A working memory workout: How to expand the focus of serial attention from one to four items in 10 hours or less. [Article]. Journal of Experimental Psychology. Learning, Memory, and Cognition, 30(6), 1322-1337.

Wadhera, D., Campanelli, L., \& Marton, K. (2018). The influence of bilingual language experience on working memory updating performance in young adults. In. T. T. Rogers, M. Rau, X. Zhu, C. W. Kalish (Eds.), Proceedings of the 40th Annual Conference of the Cognitive Science Society, 2639-2644, Madison, WI. ISBN: 978-0-9911967-8-4

Westerberg, H., Jacobaeus, H., Hirvikoski, T., Clevberger, P., Ostensson, M. L., Bartfai, A., \& Klingberg, T. (2007). Computerized working memory training after stroke - A pilot study. Brain Injury, 21(1), $21-29$ 